\title{
Divisibility Problem for one relator Monoids
}

\author{
S.I.Adian \\ Steklov Mathematical Institute \\ Gubkina str. 8, 117966 Moscow, RUSSIA \\ e-mail: si@adian.mian.su
}

We consider monoids presented by one defining relation in 2 generators:

$$
M=<a, b ; a U=b V>.
$$

Denote $A_{1}=a U$ and $A_{2}=b V$.

We say that the word $X$ is left side divisible by $Y$ in $M$ if there exists a word $Z$ such that $X=Y Z$ in $M$. The left side divisibility problem for $M$ is the requirement to find an algorithm to recognize for any two words $X$ and $Y$, whether or not $X$ is left side divisible by $Y$ in $M$ ?

The following theorem was proved in $[1,2,3]$.

Theorem 1 The word problem for any 1-relator monoids can be reduced to the left side divisibility problem for monoids $M$ presented in 2 generators by 1 defining relation of the form $a U=b V$. For the solution of this problem it sufficies to find an algorithm to recognize for any word $a W$ (or for any word $b W)$ whether or not it is left side divisible in $M$ by the letter $b$ (accordingly by the letter a). 


\begin{abstract}
Algorithm $\mathfrak{A}$
The algorithm $\mathfrak{A}$ was introduced in [2] for a more general case of monoids presented by any cyclefree system of relations. Here we shall apply this algorithm to the case of the monoid $M$.

The algorithm $\mathfrak{A}$ was used in several papers for a solution of the left side divisibility problem for monoids $M$ under some additional conditions.

To apply this algorithm one should find another algorithm $\mathfrak{B}$ that decides for any word $a W$, whether or not the algorithm $\mathfrak{A}$ terminates when applied to $a W$.

For the given word $a W$ the algorithm $\mathfrak{A}$ finds the uniquely defined prefix decomposition which is either of the form

$$
a W=P_{1} P_{2} \ldots P_{k} P_{k+1},
$$

where each $P_{i}$ is the maximal nonempty proper common prefix of the word $P_{i} P_{i+1} \ldots P_{k+1}$ and the appropriate relator $a U$ or $b V$, or of the form

$$
a W=P_{1} P_{2} \ldots P_{k} A_{j_{k}} W_{k+1},
$$

where the prefixes $P_{i}$ are defined in a similar way, but the segment $A_{j_{k}}$ is one of the relators of the monoid $M$. We call the segment $A_{j_{k}}$ the head of the decomposition (3).

Let us describe in details how our algorithm $\mathfrak{A}$ works.

Suppose we have an initial word $a W$. Consider the Maximal Common Prefix of two words $a W$ and $A_{1}$ and denote it by

$$
P_{1}=\operatorname{MCP}\left(a W, A_{1}\right) .
$$

We have $a W=P_{1} W_{1}$ and $a U=P_{1} U_{1}$ for some $W_{1}$ and $U_{1}$. 
Clearly $P_{1}$ is not empty. We consider the following 2 cases.

Case 1. If $U_{1}$ is empty, then $a W=a U W_{1}$. So we have a prefix decomposition of the form (3) for $k=0$.

In this case the algorithm $\mathfrak{A}$ replaces in $a W$ the segment $a U$ by $b V$. So we obtain $a W=b V W_{1}$ in $M$. Hence $a W$ is left side divisible by $b$ in $M$.

Case 2. Let $U_{1}$ be not empty. Then $P_{1}$ is a proper prefix of $a U$.

If $W_{1}$ is empty then $a W$ is a proper segment of the relator $a U$. It is easy to prove that the proper segment $P_{1}$ of $a U$ is not divisible by $b$ in $M$.

Hence we can assume that $U_{1}$ and $W_{1}$ both are nonempty. It follows from (4) that in this case they have different initial letters $a$ and $b$.

In this case to prolong the prefix $P_{1}$ of $a U$ in $P_{1} W_{1}$ to the right side we should divide $W_{1}$ by $b$ if it starts by $a$ or divide $W_{1}$ by $a$ if it starts by $b$. So the situation is similar to the initial one.

Now in a similar way we consider the nonempty word $P_{2}=$ $\operatorname{MCP}\left(W_{1}, A_{j}\right)$, where $A_{j_{1}}$ is the relator of $M$ which has a common initial letter with $W_{1}$.

Suppose $W_{1}=P_{2} W_{2}$ and $A_{j}=P_{2} U_{2}$. Then again we consider 2 cases.

Case 2.1. If $U_{2}$ is empty, then $W_{1}=A_{j} W_{1}$.

In this case we have the following prefix decomposition of the word $a W$ :

$$
a W=P_{1} A_{j_{1}} W_{2},
$$

where $A_{j_{1}}$ is called the head.

Case 2.2. Let $U_{2}$ be nonempty. 
In this case if $W_{2}$ is empty then $a W=P_{1} P_{2}$ where $P_{2}$ is a proper segment of of the relator $A_{j_{1}}$. Hence we obtained for $a W$ a prefix decomposition of the form (2). It is easy to prove that the word $P_{1} P_{2}$ is not divisible by $b$ in $M$.

Hence we can assume that $U_{2}$ and $W_{2}$ both are nonempty. It follows from (4) that in this case they have different initial letters $a$ and $b$.

In this case to prolong the prefix $P_{2}$ of $A_{j_{1}}$ in $P_{1} P_{2} W_{2}$ we should divide $W_{2}$ by $b$ if it starts by $a$, or divide $W_{2}$ by $a$ if it starts by $b$. So the situation again is similar to the initial one.

Hence we can consider the nonempty word $P_{3}=\operatorname{MCP}\left(W_{2}\right.$, $A_{j_{2}}$ ), where $A_{j_{2}}$ is one of the relators of $M$ which has the common initial letter with the word $W_{2}$. And so on. The length of the word $W_{i}$ is decreasing. So after a finite number of steps either we shall find some prefix decomposition of the form (3) with the head $A_{j_{k}}$ or we shall stop on some decomposition of the form (2).

It is easy to prove that if the decomposition of $a W$ is of the form (2), then the word $a W$ in $M$ is not left side divisible by $b$.

If the decomposition is of the form (3), then the algorithm $\mathfrak{A}$ replaces the head $A_{i}$ in $a W$ by the another relator in (1): $a U$ should be replaced by $b V$ or $b V$ by $a U$. Hence we get one of the following elementary transformations in the monoid $M$ :

$$
a W=P_{1} P_{2} \ldots P_{k} a U W_{k+1} \rightarrow P_{1} P_{2} \ldots P_{k} b V W_{k+1}=W^{\prime}
$$

or

$$
a W=P_{1} P_{2} \ldots P_{k} b V W_{k+1} \rightarrow P_{1} P_{2} \ldots P_{k} a U W_{k+1}=W^{\prime} .
$$

Clearly the result $W^{\prime}$ of this transformation is equal to $a W$ in $M$. If the resulting word $W^{\prime}$ starts by the letter $b$ (this happens 
only if $k=0$ !), then the algorithm $\mathfrak{A}$ terminates by the positive answer. Otherwise the algorithm $\mathfrak{A}$ repeats the same procedure with the word $W^{\prime}$.

Theorem 2 (see [2]) If the word $a W$ is left side divisible by $b$ in $M$ then the algorithm $\mathfrak{A}(a W)$ terminates with the positive result, and in this case we obtain the shortest proof of the left side divisibility of the word $a W$ by $b$ in $M$.

Conjecture 1 There exists an algorithm $\mathfrak{B}$ that decides for any word $a W$ whether or not the algorithm $\mathfrak{A}(a W)$ terminates.

Problem 1 Check if the conjecture 1 is true.

\section{REFERENCES}

1. Adian S.I. (1966). Defining relations and algorithmic problems for groups and semigroups. Proc. Steklov Inst. Math. 85. (English version published by the American Mathematical Society, 1967).

2. Adian S.I. (1976). Word transformations in a semigroup that is given by a system of defining relations. Algebra i Logika 15, 611-621; English transl. in Algebra and Logic 15 (1976).

3. Adian S.I. and Oganesian G.U. (1987). On the word and divisibility problems in semigroups with one defining relation. Mat. Zametki 41, 412-421; English transl. in Math. Notes 41 (1987).

4. Adian S.I. and V.G.Durnev. Decision problems for groups and semigroups. In "Russian Math. Surveys" (Uspechi Mat. Nauk, 2000), vol. 55, No. 2, pp. 207-296. 